\title{
Nanoarrays of tethered lipid bilayer rafts on poly(vinyl alcohol) hydrogels $\dagger$
}

\author{
Bong Kuk Lee, ${ }^{a}$ Hea Yeon Lee, ${ }^{* a}$ Pilnam Kim, ${ }^{b}$ Kahp Y. Suh ${ }^{b}$ and Tomoji Kawai ${ }^{* a}$ \\ Received 9th June 2008, Accepted 2nd September 2008 \\ First published as an Advance Article on the web 22nd October 2008 \\ DOI: 10.1039/b809732a
}

Lipid rafts are cholesterol- and sphingolipid-rich domains that function as platforms for signal transduction and other cellular processes. Tethered lipid bilayers have been proposed as a promising model to describe the structure and function of cell membranes. We report a nano(submicro) array of tethered lipid bilayer raft membranes (tLBRMs) comprising a biosensing platform. Poly(vinyl alcohol) (PVA) hydrogel was directly patterned onto a solid substrate, using ultraviolet-nanoimprint lithography (UV-NIL), as an inert barrier to prevent biofouling. The robust structures of the nanopatterned PVA hydrogel were stable for up to three weeks in phosphate-buffered saline solution despite significant swelling (100\% in height) by hydration. The PVA hydrogel strongly restricted the adhesion of vesicles, resulting in an array of highly selective hydrogel nanowells. tLBRMs were not formed by direct vesicle fusion, although raft vesicles containing poly(ethylene glycol) lipopolymer were selectively immobilized on gold substrates patterned with PVA hydrogel. The deposition of tLBRM nano(submicro) arrays was accomplished by a mixed, self-assembled monolayer-assisted vesicle fusion method. The monolayer was composed of a mixture of 2-mercaptoethanol and poly(ethylene glycol) lipopolymer, which promoted vesicle rupture. These results suggest that the fabrication of inert nanostructures and the site-selective modification of solid surfaces to induce vesicle rupture may be essential in the construction of tLBRM nano(submicro) arrays using stepwise self-assembly.

\section{Introduction}

The lipid "raft" hypothesis proposes that different lipids found in plasma membranes have different biophysical propensities to associate with each other. ${ }^{1,2}$ Lipid rafts are defined as phasesegregated domains enriched in cholesterol, sphingolipids, and certain proteins. ${ }^{1,2}$ It has been suggested that lipid rafts play a role in a wide range of biological processes, such as signal transduction pathways, apoptosis, cell adhesion and migration, and protein sorting. ${ }^{3}$ In addition to normal cellular functions, it has also been suggested that lipid rafts serve as functional hotspots for bacteria, viruses and toxins, as well as providing a microenvironment for prion formation and amyloid aggregation. ${ }^{4}$ Phase-separated domains in lipid bilayers can range in size from nanoscale to microscale, ${ }^{6}$ depending on the lipid mixture. Lipid membranes with well-defined domains may be useful for the study of lipid raft dynamics, transmembrane proteins, membrane-associated proteins, and for the realization of biological interconnections and building blocks in nanodevices or nanochips. However, a model lipid membrane system that has

${ }^{a}$ The Institute of Scientific and Industrial Research (ISIR), Osaka University, 8-1 Mihogaoka, Ibaraki, Osaka, 567-0047, Japan. E-mail: hylee@sanken.osaka-u.ac.jp; kawai@sanken.osaka-u.ac.jp; Fax: +81-66875-2440; Tel: +81-6-6879-8447

${ }^{b}$ School of Mechanical and Aerospace Engineering, Seoul National University, Seoul, 151-742, Korea

$\uparrow$ Electronic supplementary information (ESI) available: Included are figures showing the initial film thickness of PVA as a function of spin-coating velocity (Fig. S1), the UV irradiation dose for curing PVA (Fig. S2), the DSC heat flow curve (Fig. S3), the AFM images of nanoimprinted PVA at stage III (Fig. S4) and the AFM images of lipid bilayer on mica (Fig. S5). See DOI: 10.1039/b809732a well-controlled domain sizes, as well as the appropriate structure necessary for lipid raft-based research has not been developed.

Lipid bilayer membranes (LBMs), such as solid-supported lipid bilayer membranes (sLBMs), ${ }^{7}$ hybrid bilayer membranes, ${ }^{8}$ polymer-cushioned lipid bilayer membranes (cLBMs), ${ }^{9}$ and tethered lipid bilayer membranes (tLBMs),${ }^{10}$ deposited on a variety of substrates, have been developed as experimental model membranes. In addition, there is a great deal of interest in the development of LBM microarrays to localize and parallelize studies on membrane functions. Due to their importance in the design of biocompatible surfaces and membrane-based research, model LBMs have been developed for nearly all fields of cellular research including studies of membrane properties, ${ }^{6}$ biosensing platforms, ${ }^{9}$ cell adhesion, ${ }^{11}$ characterization of membrane-associated proteins, ${ }^{12}$ and drug discovery. ${ }^{13,14}$ Among various model membranes, microarrays of sLBMs have been widely investigated. To facilitate the formation of sLBM arrays, materials, such as metals and metal oxides, ${ }^{15}$ diacetylene lipids, ${ }^{16}$ polyethylene glycol (PEG)-copolymer, ${ }^{17}$ and proteins, ${ }^{18}$ have been used as patterned barriers on solid supports. Patterning methods include photolithography, ${ }^{15}$ deep-UV illumination, ${ }^{16}$ capillary molding, ${ }^{17}$ microcontact printing, ${ }^{18}$ and polymer lift-off. ${ }^{11}$ However, it has been reported that the membrane-substrate distance (5-20 $)^{19}$ of sLBMs is usually not sufficiently large to avoid direct contact between transmembrane proteins incorporated in the membrane and the solid surface. Previous studies have suggested that delamination between the membrane and the solid substrate using soft polymeric materials, such as a polymer "cushion" ", 20 or polymer "tethers," ${ }^{, 0-22}$ could reduce the risk of protein denaturation by contact with the solid substrates. Both cLBMs and tLBMs are considered promising architectural 
models that mimic the structure and function of natural biomembranes, including the formation of lipid rafts. However, there have been relatively few studies on $\mathrm{CLBM}^{23-25}$ and $\mathrm{tLBM}^{26}$ arrays. Moreover, the arrays of cLBMs and tLBMs have remained on the microscale, and there have been no other studies on nanoarrays of raft-forming LBMs in the literature.

The aim of this study was to create a nanoarray of tLBRMs on patterned substrates, to generate a membrane-based biosensing platform. For this purpose, the patterns must be scaled down from microscale to nanoscale. Nanoimprint lithography (NIL) is a simple, low-cost, and high-resolution nanopatterning method. ${ }^{27,28}$ We have previously reported the nanoarray of protein $^{29}$ and single liposome $^{30}$ with a nanopatterned PEG at the $100 \mathrm{~nm}$ scale was fabricated by ultraviolet (UV)-NIL and soft lithography, respectively. These results suggest that the direct fabrication of the nanopatterns with an inert material to protect against biofouling significantly simplified both the patterning process and the selfassembled nano(submicro) array of biomolecules.

Here, we report a very effective and widely applicable method for constructing a nanoarray of tLBRMs by using NIL. UVcurable poly(vinyl alcohol) (PVA) was used as an inert material because it minimizes protein adsorption and cell adhesion. ${ }^{31,32}$ The nanopatterns of PVA hydrogel, that is cross-linked into the swollen polymer network but does not dissolve in water, was fabricated by thermal-assisted UV-NIL (Scheme 1a). Morphological changes and stability of the PVA hydrogel nanostructures in aqueous solution were determined by atomic force microscopy (AFM). This technique was also used to demonstrate the fabrication of tLBRM nanoarrays with nanoimprinted PVA hydrogel (Scheme 1b). A direct vesicle fusion method, ${ }^{7,33}$ and a mixed, self-assembled monolayer (SAM)-assisted vesicle fusion method, ${ }^{21,34,35}$ were carried out to construct a tLBRM nanoarray within the nanostructured barriers of the PVA hydrogel.

\section{Materials and methods}

\section{Materials}

UV-curable PVA (AWP: solid content $6 \mathrm{wt} \%$ ) was provided by Toyo Gosei Kogyo Co. (Chiba, Japan). Optool DSX (20\% perfluorinated compounds) and Demnum solvent $(80 \%$ perfluoroisohexane) were obtained from Daikin Industries (Osaka, Japan). Sphingomyelin (SM), cholesterol, 1-palmitoyl2-oleoyl-sn-glycero-3-phosphocholine (POPC), and 1,2-distearoyl-sn-glycero-3-phosphoethanolamine- $N$-poly(ethylene glycol)-2000- $N$-[3-(2-(pyridyldithio)propionate]) (DSPE-PEGPDP) were purchased from Avanti Polar Lipids Inc. (Alabaster, AL, USA). Texas-Red 1,2-dihexadacanoyl-sn-glucero-phosphoethanolamine (TR-DHPE) was purchased from Molecular Probes (Eugene, OR, USA). 2-Mercaptoethanol was purchased from Sigma-Aldrich (St. Louis, MO USA) and used without further purification.

\section{Thermal-assisted ultraviolet-nanoimprint lithography (UV-NIL)}

Gold $(\mathrm{Au})$ substrates were prepared by sputtering high-purity gold $(99.999 \%)$ onto cleaned $\mathrm{SiO}_{2}$ wafers with a titanium (Ti) adhesion layer (100 nm Au and $5 \mathrm{~nm} \mathrm{Ti}$ ). The substrates were cleaned with UV-ozone for 30 min with an ozone cleaner (NLUV253; Nippon Laser Denshi, Tokyo, Japan). To fabricate the

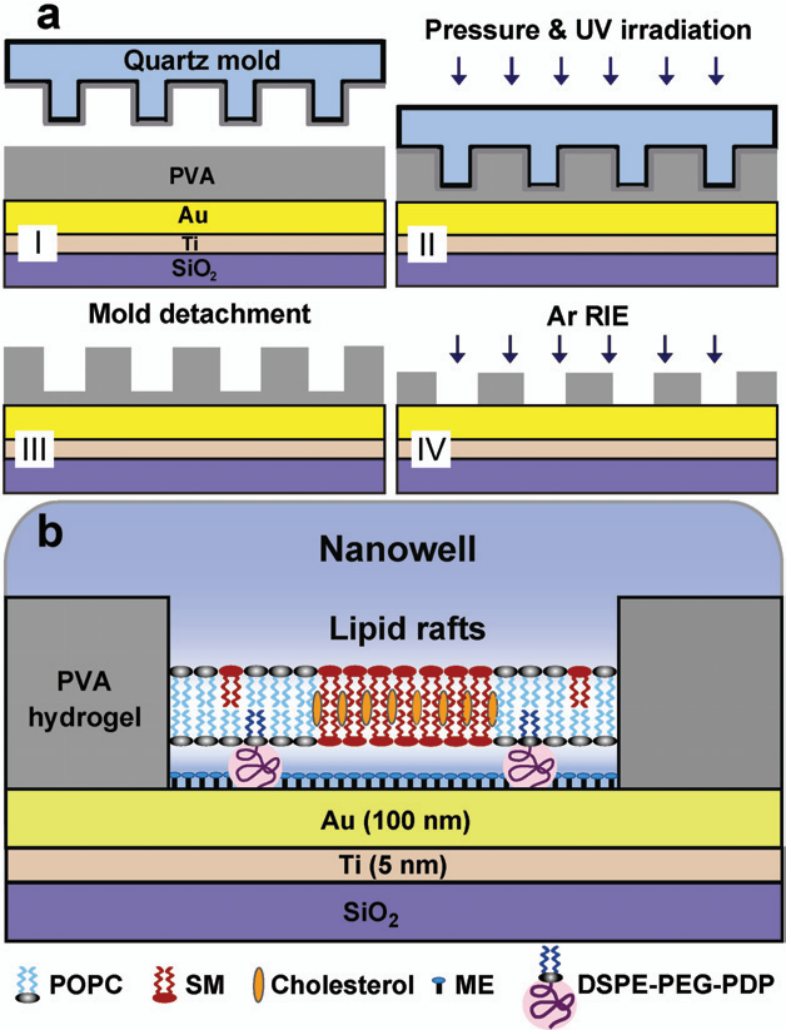

Scheme 1 (a) Schematic diagram showing the fabrication of PVA nanostructures with thermal-assisted UV-NIL. (b) Schematic diagram of the proposed morphology of a lipid raft as a form of tethered lipid bilayer membrane (tLBRM) in the nanopatterned PVA hydrogel on a gold substrate $(\mathrm{Au})$. The raft membrane, comprised of a mixture of $\mathrm{POPCl}$ $\mathrm{SM} /$ cholesterol (1: $1: 1$ molar ratio), was formed on a mixed SAM of 2-mercaptoethanol (ME) and PEG lipopolymer (DSPE-PEG-PDP) in the PVA nanowell.

nanostructure of PVA hydrogel, thermal-assisted UV-NIL was carried out using an instrument from Nanoimprinter Systems (NM-401; Meisyo Kiko, Hyogo, Japan) equipped with a UV lamp (Toscure251; Toshiba, Tokyo, Japan) (Scheme 1a). The substrates were then spin-coated with a thin film of UV-curable PVA, followed by prebaking at $50^{\circ} \mathrm{C}$ for $5 \mathrm{~min}$. The PVA-coated substrates were heated to above the glass transition temperature ( $T_{\mathrm{g}}: 40.5^{\circ} \mathrm{C}$ ) of PVA. A positive quartz mold (100 nm in height), coated with $0.1 \mathrm{wt} \%$ Optool DSX as a release agent to prevent adhesion of cured resist to the mold, was then pressed for 5 min at an imprint pressure of $2 \mathrm{MPa}$ at $80{ }^{\circ} \mathrm{C}$ under vacuum. After cooling to room temperature while maintaining the pressure, the PVA was cured using UV irradiation (wavelength, $365 \mathrm{~nm}$; dose, $\left.100 \mathrm{~mJ} \mathrm{~cm} \mathrm{~cm}^{-2} \mathrm{UV}\right)$. The mold was then removed from the substrate. Residual PVA was subsequently removed by argon reactive ion etching (RIE: Ar gas flow $=10 \mathrm{sccm}$, pressure $=4$ $\mathrm{Pa}$, power $=50 \mathrm{~W}$ ) using an RIE system (RIE-10NR; Samco Inc., Tokyo, Japan). The etching time was varied to control the depth of the nanowells.

\section{Swelling measurements of PVA hydrogel}

Dry hydrogel samples, prepared by UV irradiation, were immersed in an excess of Milli-Q deionized water or $10 \mathrm{mM}$ 
phosphate-buffered saline (PBS) at room temperature. At specific time intervals, the samples were removed from the water or $10 \mathrm{mM}$ PBS and excess surface water was dried with filter paper. The swollen mass $\left(\mathrm{W}_{\mathrm{s}}\right)$ was weighed until the hydrated gels reached a constant weight. The hydrogel samples were subsequently dried for $48 \mathrm{~h}$ in desiccators at room temperature and their dry weights $\left(\mathrm{W}_{\mathrm{d}}\right)$ were recorded.

\section{Lipid vesicle preparation}

Model raft vesicles ${ }^{5,36}$ were prepared by the extrusion method. Briefly, a lipid mixture of POPC, SM, and cholesterol (1:1 : 1 molar ratio), with or without $5 \mathrm{~mol} \%$ DSPE-PEG-PDP, was dissolved in chloroform and mixed in a round-bottomed flask. The organic solvent was evaporated using a rotary evaporator (RE 440; Yamato Scientific Co., Ltd., Tokyo, Japan) at $45^{\circ} \mathrm{C}$ in a water bath and vacuum-desiccated overnight. The dry lipid film was hydrated in $10 \mathrm{mM}$ PBS at $45^{\circ} \mathrm{C}$ and vortexed for $30 \mathrm{~min}$ to generate multilamellar vesicles. The resulting vesicles were freezethawed five times to prepare the unilamellar vesicles. Subsequently, uniformly sized vesicles were obtained by extrusion of the unilamellar vesicles through polycarbonate filters with a pore diameter of $100 \mathrm{~nm}$ in an extrusion apparatus (Avestin Inc., Ottawa, ON, Canada). Vesicle size and PEG lipopolymer dimensions were confirmed by non-invasive back-scattering method in $10 \mathrm{mM}$ PBS (Zetasizer Nano ZS; Malvern Instruments Ltd., Malvern, Worcestershire, UK).

\section{Construction of tBLRM nanoarray}

Two methods were used to confine the tBLRM to the nanopatterned PVA hydrogel: a vesicle fusion method and a mixed self-assembled monolayer (SAM)-assisted vesicle fusion method. For a vesicle fusion method, a few drops of raft vesicles containing $5 \mathrm{~mol} \%$ DSPE-PEG-PDP were evenly distributed onto the patterned PVA hydrogel and incubated at room temperature for $1 \mathrm{~h}$, and then the sample was rinsed thoroughly with PBS. For a mixed SAM-assisted vesicle fusion method, the mixtures of $1 \mathrm{mM}$ DSPE-PEG-PDP and $9 \mathrm{mM}$ ME were dissolved in $10 \mathrm{mM}$ PBS buffer. One-step mixed SAM was prepared by immersing the PVA patterned gold substrates in the mixtures of DSPEPEG-PDP/ME at room temperature for $2 \mathrm{~h}$ and the sample was rinsed with PBS several times to remove excess molecules. Subsequently, a model raft vesicles comprising a mixture of POPC/SM/cholesterol (1 $: 1: 1$ molar ratio) was dropped on PVA patterned gold substrate modified with a mixed SAM. After $2 \mathrm{~h}$ at room temperature, the excess unfused vesicles were flushed out of the PVA patterned gold substrates. To confirm the formation of single lipid bilayer on the mixed SAM, the micropattern of the DSPE-PEG-PDP/ME mixed SAM was fabricated on gold substrate by combining polymer lift-off and molecular assembly. For the polymer lift-off, a thin film of $125 \mathrm{~nm}$ of the poly(methyl methacrylate) (PMMA; MicroChem Corp., Newton, MA, USA) was imprinted at the temperature of $150{ }^{\circ} \mathrm{C}$ and pressure of $5 \mathrm{MPa}$ for $5 \mathrm{~min}$ after prebaked at $80^{\circ} \mathrm{C}$ for $5 \mathrm{~min}$. After expose the gold surface by Ar RIE, the substrates patterned with PMMA was modified with the mixed SAM for $2 \mathrm{~h}$, followed by the PMMA lift-off by the sonication in acetone at $45^{\circ} \mathrm{C}$ for $1 \mathrm{~h}$. The sample was then rinsed with excess Milli-Q several times.
The formation of lipid bilayer on the patterned mixed SAM was carried out by the addition of a few drops of $1 \mathrm{mM}$ raft vesicles.

\section{Fluorescence microscopy}

Fluorescence microscopy was performed using an Olympus BX51 inverted research microscope equipped with a fluorescence attachment (IX-FLA; Olympus, Tokyo, Japan) and a highresolution digital camera (DP70; Olympus) for image acquisition. Red emission light (>590 nm) was filtered using a U-MWG Olympus filter cube.

\section{Atomic force microscopy (AFM)}

To image the patterned hydrogel and lipid bilayers, we used a Digital Instruments NanoScope III atomic force microscope (Veeco Instruments Inc., Woodbury, NY, USA) in tapping mode in both air and aqueous phases at ambient temperature. The scan rate was $0.5 \mathrm{~Hz}$ and 512 lines were scanned per sample. AFM imaging in air was carried out with a silicon cantilever with a nominal spring constant of $2 \mathrm{~N} \mathrm{~m}^{-1}$ (Olympus). AFM imaging in aqueous solutions was carried out with the aid of a fluid cell and a V-shaped silicon nitride cantilever with a nominal spring constant of $0.58 \mathrm{~N} \mathrm{~m}^{-1}$ (Veeco Metrology Group, Tucson, AZ, USA). The imaging force was minimized to limit deformation of the hydrogel by the AFM cantilever. Data were processed using SPIP V3.3.7.0 software (Image Metrology, Lyngby, Denmark).

\section{Results and discussion}

\section{Nanopatterning of PVA hydrogel using UV-NIL}

The effects of initial film thickness, glass transition temperature $\left(T_{\mathrm{g}}\right)$, and UV irradiation dose were investigated to optimize the NIL process using PVA. Based on the results of these investigations, a film of PVA $137 \mathrm{~nm}$ thick was spin-coated onto gold or $\mathrm{SiO}_{2}$ substrates (Figure $\mathrm{S} 1, \mathrm{ESI}$ ). $\dagger$ The UV irradiation dose for curing the PVA and the imprinting temperature were $100 \mathrm{~mJ}$ $\mathrm{cm}^{-2}$ and $80{ }^{\circ} \mathrm{C}$ above $T_{\mathrm{g}}\left(40.5^{\circ} \mathrm{C}\right)$ of PVA, respectively (Figure S2 and S3, ESI). $\dagger$ The nano(submicron) patterns of PVA hydrogel was fabricated by thermal-assisted UV-NIL process as shown in Scheme 1a.

Fig. 1 shows the height and cross-sectional atomic force microscopy (AFM) images of the $500 \mathrm{~nm}$ patterns of the dry PVA hydrogel on a gold substrate before and after argon (Ar) reactive ion etching (RIE). When the mold was removed from the substrates (Fig. 1a and 1c), the depth of the imprinted PVA patterns was the same height $(100 \mathrm{~nm})$ as the mold under thermal-assisted UV-NIL conditions (Fig. 1a and 1c). The micro/ nanopatterns of the PVA hydrogel with the feature size down to $100 \mathrm{~nm}$ were successfully fabricated through thermal-assisted UV-NIL (Figure S4, ESI). $\dagger$ These indicated that the mold patterns were faithfully transferred by the imprinting technique. To expose the gold substrate and control the height of the patterned PVA hydrogel features, the Ar RIE was subsequently performed. Height and cross-sectional AFM images shown in Fig. $1 \mathrm{~b}$ and $1 \mathrm{~d}$ indicate that the substrate surface was completely exposed with good edge definition when removed by Ar RIE (gas flow $=10 \mathrm{sccm}$, pressure $=4 \mathrm{~Pa}$, power $=50 \mathrm{~W}$ ). The etching rate of dry PVA was about $34 \mathrm{~nm} \mathrm{~min}^{-1}$. 


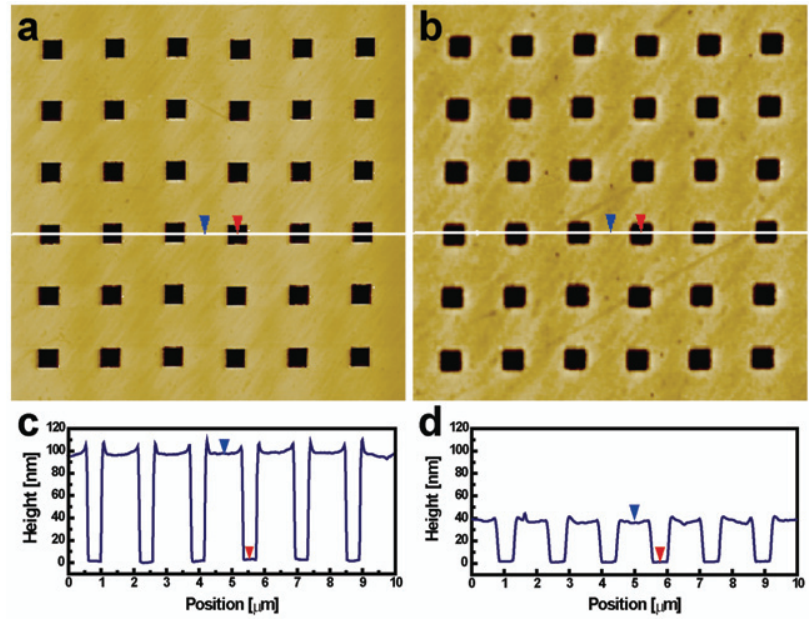

Fig. 1 (a, b) Height and (c, d) cross-sectional AFM images of the $500 \mathrm{~nm}$ patterned PVA hydrogel on gold substrate (a, c) before and (b, d) after Ar RIE (gas flow $=10 \mathrm{sccm}$, pressure $=4 \mathrm{~Pa}$, power $=50 \mathrm{~W}$ ).

\section{Swelling behavior of PVA hydrogel}

The swelling behavior of a hydrogel is important, especially in characterizing morphological changes. The swelling ratio $\left(Q_{\mathrm{r}}\right)$ of $3 \mathrm{wt} \%$ PVA was calculated according to following equation:

$$
Q_{\mathrm{r}}=\left(W_{\mathrm{s}}-W_{\mathrm{d}}\right) / W_{\mathrm{d}}
$$

Here, $W_{\mathrm{s}}$ and $W_{\mathrm{d}}$ are the weights of the swollen and dry sample, respectively.

The $Q_{\mathrm{r}}$ values of $3 \mathrm{wt} \%$ PVA hydrogel in phosphate-buffered saline solution (10 mM PBS, $\mathrm{pH}$ 7.4), and Milli-Q deionized water, were equilibrated for 2 and 3 arbitrary units for $30 \mathrm{~min}$ at room temperature, respectively (Fig. 2a). Based on these observations, only PBS was used and the samples were not exposed to air until AFM measurements were completed. To investigate the morphological changes of the nanopatterned PVA hydrogel induced by hydration, the gold substrates patterned with PVA hydrogel, with a mean feature height of $58 \mathrm{~nm}$, were incubated in $10 \mathrm{mM}$ PBS for $1 \mathrm{~h}$. As shown in Fig. 2c and 3d, the mean height of the hydrated PVA pattern increased by about twofold compared to that of the dry PVA (Fig. 2b). Interestingly, a lateral swelling was not observed within the resolution constraints of the AFM measurements. This result indicates that the swelling of PVA hydrogel is highly anisotropic with little change in the lateral dimensions presumably as a result of the constraints imposed by the binding of the hydrogel to the substrate surface. Similar swelling phenomenon for the nanopatterns of PEG hydrogel have been observed in our previous study. ${ }^{29}$ The nano(submicro) patterned PVA hydrogel maintained these robust structures on the gold surface for at least 3 weeks in PBS, even though the features were swollen by hydration, and remained intact in ethanol and acetone for 2 months.

\section{Site-selective confinement of raft vesicles}

The feasibility of creating a selective array of vesicles on patterned PVA hydrogel was confirmed by fluorescence microscopy. The micro/nanopatterns of the PVA hydrogel were
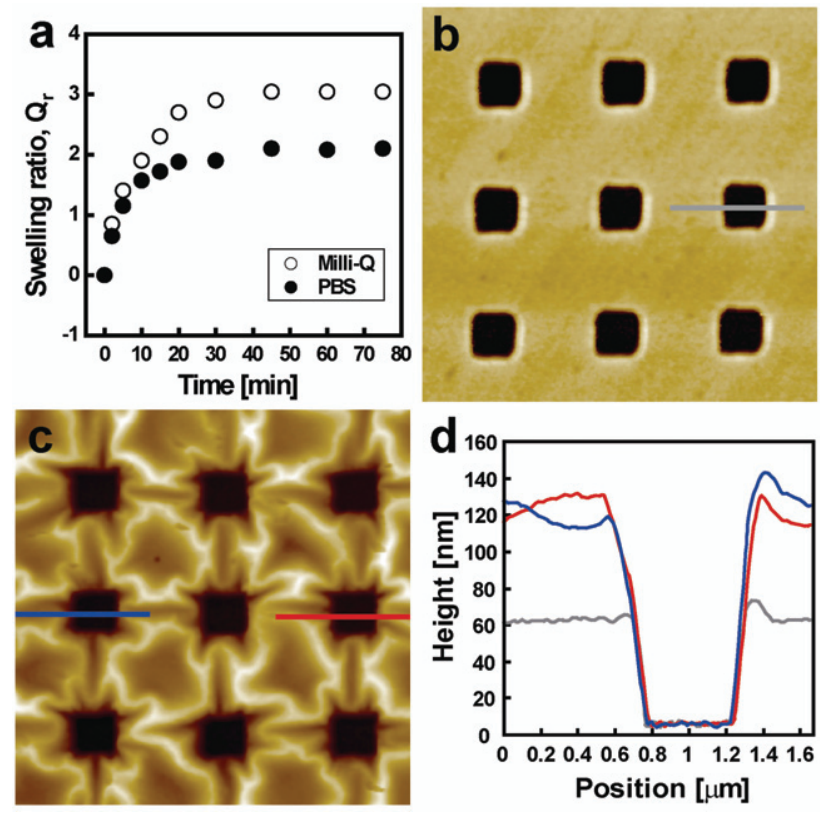

Fig. 2 (a) Swelling behavior of PVA hydrogel in $10 \mathrm{mM}$ PBS and deionized water at room temperature. Dry patterns with a mean height of $58 \mathrm{~nm}$ were used. (b, c) Height AFM images of the $500 \mathrm{~nm}$ patterned PVA hydrogels on gold substrate (b) before and (c) after swelling by $10 \mathrm{mM}$ PBS solution. (d) Cross-sectional AFM images at each sate: (gray line) dry state and (blue and red lines) hydrated state.

fabricated on $\mathrm{SiO}_{2}$ instead of gold substrates due to the quenching effects of the gold substrate.

Fig. 3a and 3b show optical images of the $1 \mu \mathrm{m}$ (a) and $500 \mathrm{~nm}$ (b) patterned PVA hydrogel on $\mathrm{SiO}_{2}$ substrates after Ar RIE (same conditions as in Fig. 1). A suspension of raft vesicles, containing $1 \mathrm{~mol} \%$ Texas-red 1,2-dihexadacanoyl-sn-glucerophosphoethanolamine (TR-DHPE), was dropped onto the patterned substrates and incubated at room temperature for $1 \mathrm{~h}$, and the sample was subsequently rinsed thoroughly with $10 \mathrm{mM}$ PBS. As shown in the TR-DHPE fluorescence images (Fig. 3c and 4d), the nonspecific adsorption of vesicles to the PVA surface was strongly restricted and the vesicles were selectively arrayed onto the patterned PVA hydrogel. These results indicate that PVA hydrogels are good, inert materials for the selective confinement of lipid vesicles and/or bilayers on solid substrates. In addition, this anti-biofouling effect of PVA may provide the site-selectivity necessary to achieve the self-assembly of biomolecules on PVA patterned substrates.

\section{Construction of tLBRM nanoarray}

Vesicle fusion method. We want to construct a nanoarray of tLBRMs on gold substrates patterned with PVA hydrogel as illustrated in Scheme 1b, because the gold substrate is used for a lot of measurement system such as surface plasmon resonance, impedance spectroscopy, and surface acoustic wave techniques. The vesicle fusion method is one of the easiest and most versatile means of forming substrate-supported LBMs. To construct a nano(submicro) array of tLBRMs on gold substrates patterned with PVA hydrogel, the direct vesicle fusion method ${ }^{7,33}$ was performed using raft vesicles containing $5 \mathrm{~mol} \%$ DSPE-PEG- 


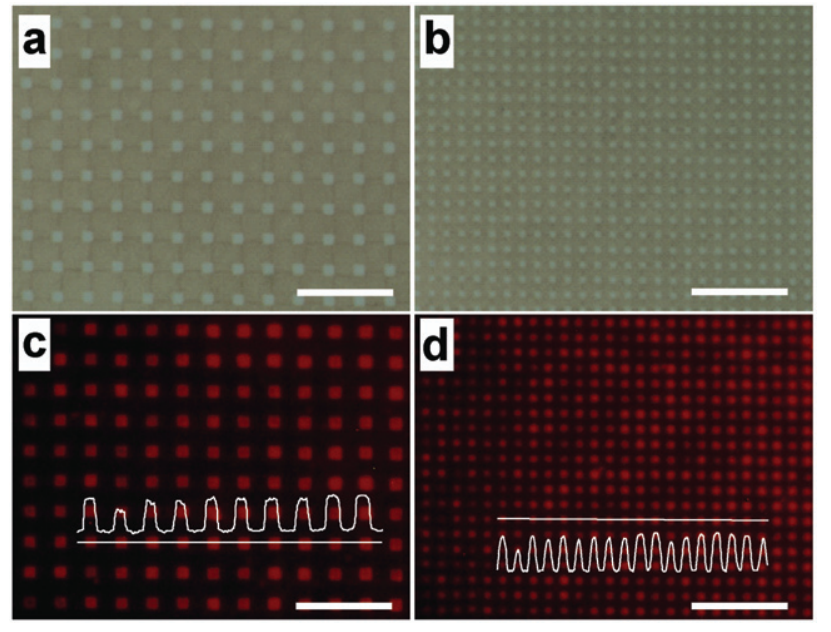

Fig. 3 (a, b) Optical and (c, d) fluorescent images of (a, c) $1 \mu \mathrm{m}$ and (b, d) $500 \mathrm{~nm}$ patterned PVA hydrogel on $\mathrm{SiO}_{2}$ substrates. Fluorescent images of raft vesicles, containing $1 \mathrm{~mol} \%$ TR-DHPE, are shown after selective deposition onto PVA patterned $\mathrm{SiO}_{2}$ substrates. Insets show the fluorescence intensity. Scale bars: $10 \mu \mathrm{m}$.

PDP, covalently bound to the gold surface via thiolate bonds. ${ }^{37}$ For this experimental approach, dry PVA patterns, with a mean feature height of $35 \mathrm{~nm}$, were used.

Fig. 4 shows the AFM images of the nano(submicron) patterns of PVA hydrogel on gold substrates after the dropwise addition of raft vesicles contanining $5 \mathrm{~mol} \%$ DSPE-PEG-PDP. The AFM height (Fig. 4a and 4d), and corresponding phase (Fig. 4b and $4 \mathrm{e})$, images clearly show that the raft vesicles were selectively immobilized in the nanowells of the PVA hydrogel, which prevented nonspecific adsorption. This result is in good agreement with the fluorescent images shown in Fig. 3. However, the raft vesicles did not rupture upon adsorption onto the gold substrates and remained spherical, with a height of 30-50 nm, for several days. These results contradict those of a previous study in which vesicles containing $5 \mathrm{~mol} \%$ PEG conjugated-phosphatidylethanolamine lipids were ruptured on glass substrates by vesicle fusion methods. ${ }^{33}$ The stability of the PVA hydrogel patterns was unaffected by the confinement of the raft vesicles. Roughness analyses were performed using SPIP V3.3.7.0 software to characterize the morphological changes in the nano(submicro) patterned PVA hydrogel by both hydration and vesicle confinement. As shown in Fig. 4c (500 nm pattern) and 4f (300 nm pattern), the surface roughness average $\left(\mathrm{S}_{\mathrm{a}}\right.$ : blue bar) of hydrated PVA increased by $17-18 \mathrm{~nm}$ compared to the dry state $\left(\mathrm{S}_{\mathrm{a}}\right.$ : gray bar). The mean height ( $\mathrm{S}_{\text {mean }}$ : blue bar) of the hydrated PVA pattern increased by about twofold compared to that of the dry PVA ( $\mathrm{S}_{\text {mean }}$ : gray bar). It can also be seen that the valley depth $\left(\mathrm{S}_{\mathrm{vk}}\right.$ : red bar) was decreased significantly compared to that of the hydrated state $\left(\mathrm{S}_{\mathrm{vk}}\right.$ : blue bar) upon selective vesicle confinement in the PVA nanowells, indicating that changes in $S_{v k}$ represent changes in the depth of the nanowells. As shown above, the direct vesicle fusion method was unsuitable for the rupture of lipid vesicles on gold substrates. ${ }^{15}$ It is generally known that vesicle rupture is induced by bilayer-substrate interactions involving electrostatic, hydration, and long-range van der Waals forces. The combination of physical forces creates an energetic

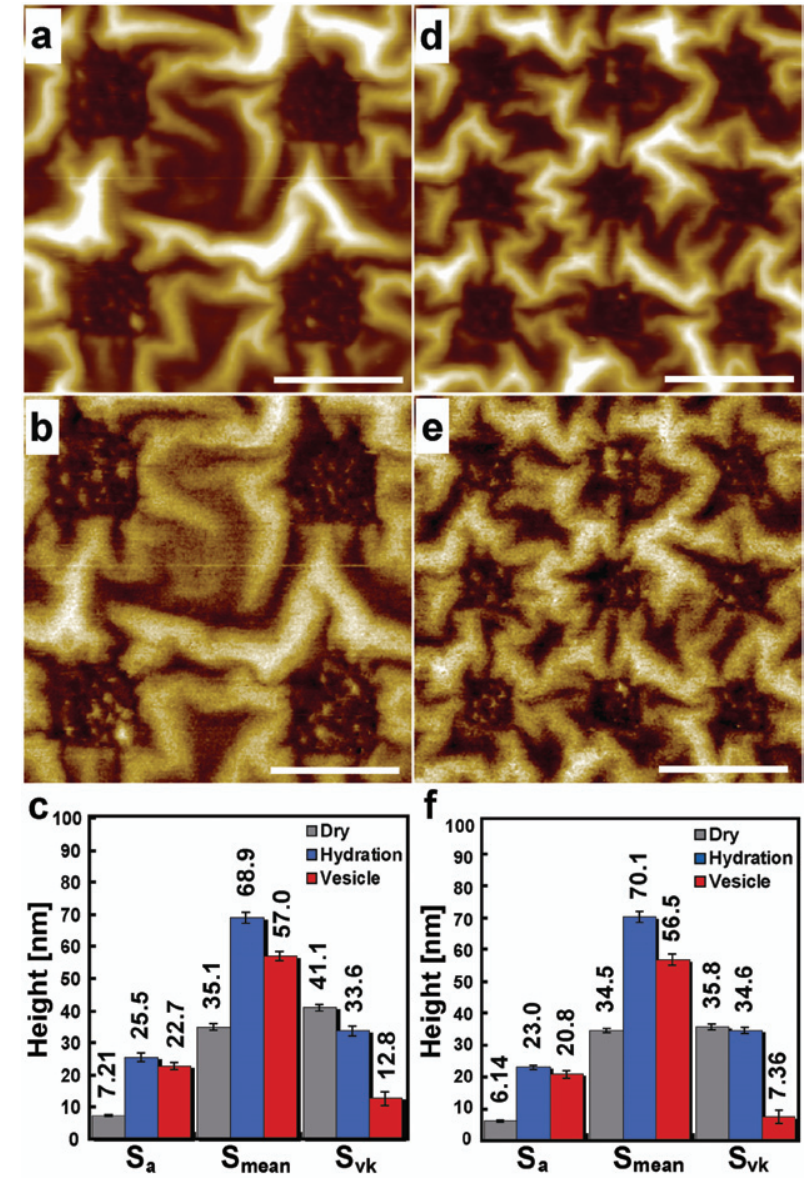

Fig. 4 (a, d) Height and (b, e) phase AFM images of (a, b) $500 \mathrm{~nm}$ and (d, e) $300 \mathrm{~nm}$ patterned PVA hydrogels on gold substrates in $10 \mathrm{mM}$ PBS after the dropwise addition of raft vesicles containing $5 \mathrm{~mol} \%$ DSPEPEG-PDP. Scale bars: $1 \mu \mathrm{m}$. Roughness charts of (c) $500 \mathrm{~nm}$ and (f) 300 nm patterns at each state: (gray bar) dry states, (blue bar) hydrated states, and (red bar) vesicle dropped state. Roughness data $\left(\mathrm{S}_{\mathrm{a}}\right.$ : roughness average, $\mathrm{S}_{\text {mean }}$ : mean height, and $\mathrm{S}_{\mathrm{vk}}$ : reduced valley height), that is the averaged values at five different locations on the same substrate, were estimated using SPIP V3.3.7.0.

minimum that tightly traps the bilayer near the surface. ${ }^{9}$ Therefore, vesicle rupture occurs easily on highly hydrophilic, solid surfaces such as glass, ${ }^{7,22}$ quartz, ${ }^{22}$ mica, ${ }^{9}$ oxidized $\mathrm{Si},{ }^{15}$ and hydrophilized gold. ${ }^{10,21,23,34,35}$ These studies suggest that vesicle rupture would be promoted if the gold substrates were made hydrophilic. Thus, vesicle fusion to a mixed, self-assembled monolayer (SAM), comprised of a mixture of PEG lipopolymer and hydrophilic molecules with thiol functional groups, ${ }^{10,23,34,35}$ may be an appealing way to construct nanoarrays of tethered lipid bilayers on gold substrates nanopatterned with the PVA hydrogel.

\section{Mixed SAM-assisted vesicle fusion method}

Based on above concept, the mixed, SAM-assisted vesicle fusion method was used to construct an array of tLBRMs into the PVA hydrogel nanowells. DSPE-PEG-PDP was used as lipopolymer tether, which exhibits minimal disruptive interactions with the bilayer and any other reconstituted membrane components. ${ }^{9}$ To 
reduce the influence of acyl chains on the lateral fluidity of bilayers and the formation of lipid rafts, DSPE-PEG-PDP was diluted 20-fold with 2-mercaptoethanol (ME) in PBS, and was used to form the mixed SAM. The gold substrates, after being nanopatterned with PVA hydrogel, were modified with a mixed SAM. The stepwise self-assembly of both the mixed SAM and the tLBRMs into the PVA nanowells was characterized by AFM to record changes in morphology and film thickness. For this experimental approach, dry PVA patterns, with a mean feature height of $45 \mathrm{~nm}$, were used.

Fig. 5 shows AFM height and phase (inset) images (a-c: $500 \mathrm{~nm}$ patterns and e-g: $300 \mathrm{~nm}$ patterns) of each step, i.e., the hydrated state (a, e), the mixed SAM-formed state (b, f), and the vesicle-dropped state (c, g). The morphologies of the DSPEPEG-PDP/ME mixed SAM-formed state and the hydrated state were similar. The presence of any adsorbate on the surface of the PVA hydrogel at the mixed SAM-formed state was not observed (Fig. 5b and 5f). Interestingly, no vesicle-like configurations were observed on the surface of the PVA hydrogel or on the inside of PVA nanowells after depositing the raft vesicle suspension; see AFM height (c, g) and corresponding phase (inset) images. Furthermore, these images differ from the vesicle-immobilized images shown in Fig. 4. There are two possible explanations for these results. First, the raft vesicles may have ruptured on the mixed SAM, forming tLBRMs as expected. Second, the raft vesicles may not have been confined on the mixed SAM in the PVA hydrogel nanowells. To verify the formation of tLBRMs, we carefully characterized the stepwise formation of both the mixed SAM and tLBMs based on the results of roughness analyses. The roughness charts in Fig. $5 \mathrm{~d}$ and $6 \mathrm{~h}$ indicates that the mean height ( $\mathrm{S}_{\text {mean }}$ : gray bar) of hydrated PVA increased by about twofold compared to the dry state $(45 \mathrm{~nm})$. This result was in good agreement with those of the analysis shown in Fig. 4. Compared with the hydrated states (a, e), the roughness average $\left(\mathrm{S}_{\mathrm{a}}\right)$ of the mixed SAM-formed $(\mathrm{b}, \mathrm{f})$, and raft vesicle-dropped states (c, g) decreased to within $4 \mathrm{~nm}$, indicating that both the DSPE-PEG-PDP/ME mixture and the raft vesicles were not markedly adsorbed and did not adhere to the surface of the PVA hydrogel, respectively. Conversely, both the mean height $\left(\mathrm{S}_{\text {mean }}\right)$ and the reduced valley height $\left(\mathrm{S}_{\mathrm{vk}}\right)$ decreased sequentially with mixed SAM formation and raft vesicle dropping. The differences in $\mathrm{S}_{\mathrm{vk}}$ between the hydrated and mixed SAM-formed states were $4.3 \mathrm{~nm}$ and $7.3 \mathrm{~nm}$ for $500 \mathrm{~nm}$ and $300 \mathrm{~nm}$ patterns, respectively. The differences in $\mathrm{S}_{\mathrm{vk}}$ between the mixed SAM-formed and vesicle-dropped states were $6.7 \mathrm{~nm}$ and $6.1 \mathrm{~nm}$ for $500 \mathrm{~nm}$ and $300 \mathrm{~nm}$ patterns, respectively. These results strongly suggest that the mixed SAM was selectively modified on the gold substrates patterned with PVA hydrogel and the raft vesicles were preferentially fused on the mixed SAM in the PVA hydrogel nanowells.

To confirm the above results, the film thicknesses of the mixed SAM and the lipid bilayer were investigated. To ascertain the thickness of the mixed SAM layer, the DSPE-PEG-PDP/ME mixed SAM was estimated using the polymer liftoff method ${ }^{29}$ and the size distribution of DSPE-PEG-PDP was investigated using a non-invasive back-scattering method in $10 \mathrm{mM}$ PBS. The thickness of the tLBRM layers, composed of POPS/SM/cholesterol with the same molar ratio, was measured using AFM on the gold substrate patterned with the mixed SAM in air.

The $1 \mu \mathrm{m}$ pattern of the DSPE-PEG-PDP/ME mixed SAM was fabricated by combining NIL and polymer liftoff (Fig. 6a and $6 \mathrm{c}$ ), and the thiol-functionalized PEG-lipopolymer and mercaptoethanol were successfully grafted onto the gold substrates. The thickness of the mixed SAM in air was $4.2 \mathrm{~nm}$ (Fig. 3c), though the poly(methyl methacrylate) (PMMA) lift-off
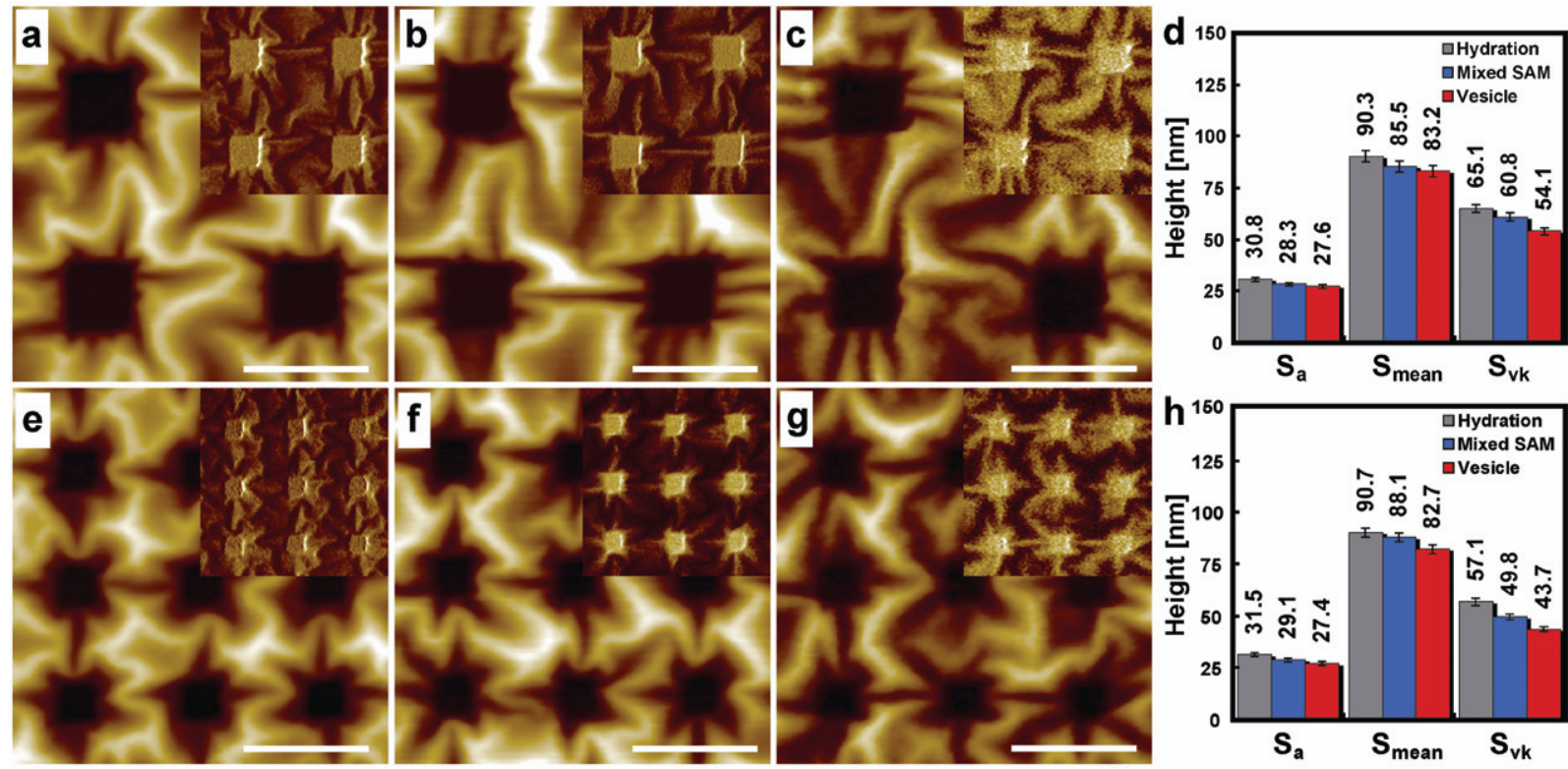

Fig. 5 Height and phase (inset) AFM images of (a-c) $500 \mathrm{~nm}$ and (e-g) $300 \mathrm{~nm}$ patterned PVA hydrogels on gold substrates in $10 \mathrm{mM}$ PBS. Dry patterns with a mean height of $45 \mathrm{~nm}$ were used: (a, e) hydrated states, (b, f) DSPE-PEG-PDP/ME mixed SAM-formed states, (c, g) raft vesicle dropped states. Scale bars: $1 \mu \mathrm{m}$. Roughness charts of (d) $500 \mathrm{~nm}$ and (h) $300 \mathrm{~nm}$ patterned PVA hydrogels at each state: (gray bar) hydrated state, (blue bar) mixed SAM-formed state, and (red bar) vesicle-dropped state. Roughness data ( $\mathrm{S}_{\mathrm{a}}$ : roughness average, $\mathrm{S}_{\mathrm{mean}}$ : mean height, $\mathrm{S}_{\mathrm{vk}}$ : reduced valley height), that is the averaged values at five different locations on the same substrate, were estimated using SPIP V3.3.7.0. 

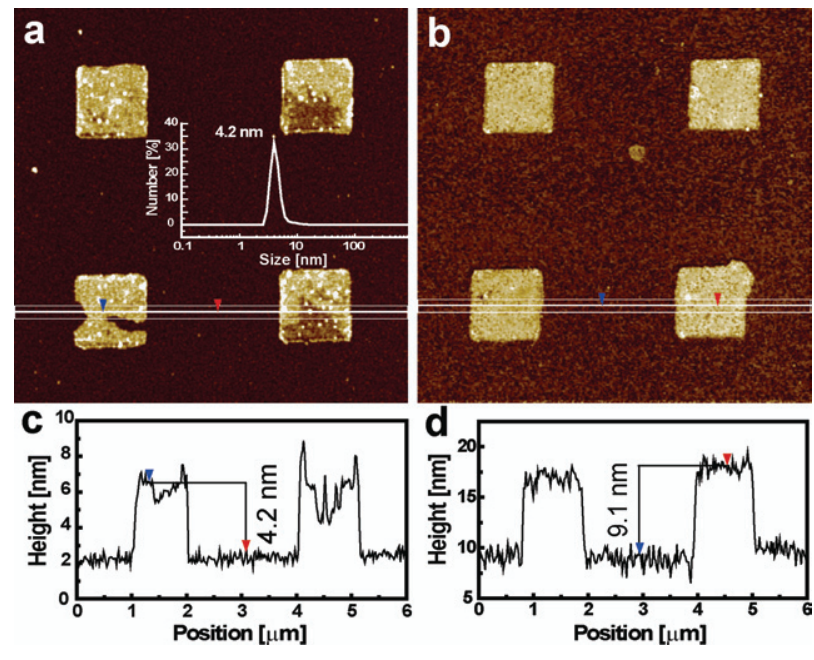

Fig. 6 (a, b) Height and (c, d) cross-sectional AFM images of a $1 \mu \mathrm{m}$ patterns of $(a, c)$ the mixed SAM-formed state after PMMA lift-off and $(b, d)$ the raft vesicle dropped state on gold substrates in air. Inset shows the size distribution of DSPE-PEG-PDP measured by non-invasive backscattering in $10 \mathrm{mM}$ PBS at room temperature.

is not complete. The size of DSPE-PEG-PDP in PBS solution was $4.2 \mathrm{~nm}$ (Inset in Fig. 6a), consistent with above result. This value was very close to the differences in $S_{\mathrm{vk}}$ values between the hydrated and mixed SAM-formed states. The differences in $\mathrm{S}_{\mathrm{vk}}$ values for $500 \mathrm{~nm}$ and $300 \mathrm{~nm}$ patterns were $4.3 \mathrm{~nm}$ and $7.3 \mathrm{~nm}$, respectively (Fig. 5). Although the differences were more pronounced in the mixed SAM-formed state on the $300 \mathrm{~nm}$ patterns due to incomplete rinsing of the sample, there is good agreement with the observations in Fig. 5. After dropping the vesicle solution, the AFM images (Fig. 6b and d) clearly show that the raft vesicles were selectively ruptured on the prepatterned mixed SAM. No vesicle-like configurations were observed on the gold substrate patterned with the mixed SAM (Fig. 6b). The thickness of lipid bilayer on the mixed SAM in air and on mica in PBS solution was estimated by $4.9 \mathrm{~nm}$ (Fig. 6b and $6 \mathrm{~d}$ ) and $5.9 \mathrm{~nm}$ (Figure S5), $\dagger$ respectively. These values are good agreement with the differences in $\mathrm{S}_{\mathrm{vk}}$ values for $500 \mathrm{~nm}$ $(6.7 \mathrm{~nm})$ and $300 \mathrm{~nm}(6.1 \mathrm{~nm})$ patterns in Fig. 5. Furthermore the tLBRMs formed in patterned PVA hydrogel are stable over 3 weeks in PBS solution. These results clearly shows that the mixed SAM composed of a hydrophilic ME and a PEG lipopolymer tether indused the vesicle rupture and the formation of the single lipid bilayer rather than the lipid multilayer. Here, the PEG lipopolymer, covalently linked to the gold substrate, penetrated into the lower leaflet of the bilayer and provided a space between the bilayer and gold substrate. The lipid rafts were nano(submicro) arrayed onto a nanoimprinted PVA hydrogel, as shown in Scheme 1b. This model system, tethered with PEG lipopolymer, will provide the required mechanical stability without losing the fluid nature of the membrane.

We described here a simple method for constructing nano(submicro) arrays of tLBRMs by combining thermal-assisted UV-NIL and stepwise molecular self-assembly. A model system, consisting of nanoarrays of tLBMs with well-defined, phasesegregated domains, was constructed by simple surface modification with a mixed SAM layer on gold substrates nanopatterned with the inert hydrogel. This system is useful for studies of transmembrane proteins, membrane-associated proteins, and lipid raft-related biosensing platforms. Although the size and density of the lipid rafts in the PVA hydrogel nanowells is not known accurately due in part to the influence of DSPE-PEGPDP in this experiment, it is known from previous research to be in the range of $75-100 \mathrm{~nm}$ for lipid vesicle systems (POPC : SM : cholesterol $=1: 1: 1$ molar ratio).$^{5}$ Nevertheless, the size of lipid rafts on mixed SAMs in nanopatterned PVA hydrogel can be controlled by varying the molar ratio of each lipid. ${ }^{5,36}$ It is believed that the success or failure of lipid raft nano(submicro) array formation is determined by the presence of an inert nanobarrier and an appropriate surface modification conducive to vesicle rupture.

\section{Conclusions}

We have developed a simple method for the construction of nano(submicro) arrays of tLBRMs in a nanopatterned PVA hydrogel on a gold substrate. Thermal-assisted UV-NIL was used to fabricate robust nanostructures of UV-curable PVA hydrogel, which acted as an inert barrier against nonspecific adsorption of raft vesicles with the same molar ratio of POPS/ $\mathrm{SM} /$ cholesterol. Two methods were used to construct these nanoarrays: traditional vesicle fusion, and a mixed SAM-assisted vesicle fusion method. The mixed SAM-assisted vesicle fusion method, using stepwise and site-selective self-assembly, was more efficient in constructing a tLBRM nanoarray with individual addressability in the nanopatterned PVA hydrogel. This tLBRM system will be widely applicable to lipid raft-related research, including cell adhesion studies, protein sorting, cell binding studies of bacteria, viruses, and toxins, and amyloid fibril formation. This platform is also amenable to high-throughput applications, such as nanodevice or nanochip development.

\section{Acknowledgements}

This work was supported by Core Research for Evolutional Science and Technology (CREST) of Japan Science and Technology Agency (JST), and New Energy and Industrial Technology Development Organization (NEDO).

\section{References}

1 K. Simons and E. Ikonen, Nature., 1997, 387, 569-572.

2 D. A. Brown and E. London, Annu. Rev. Cell Dev. Biol., 1998, 14, 111-136.

3 K. Simons and D. Toomre, Nat. Rev. Mol. Cell Biol., 2000, 1, 31-39.

4 J. Fantini, N. Garmy, R. Mahfoud and N. Yahi, Exp. Rev. Mol. Med., 2002, 2002, 1-22.

5 R. F. M. de Almeida, L. Loura, A. Fedorov and M. Prieto, J. Mol. Biol., 2005, 346, 1109-1120.

6 C. Dietrich, L. A. Bagatolli, Z. N. Volovyk, N. L. Thompson, M. Levi, K. Jacobson and E. Gratton, Biophys. J., 2001, 80, 14171428.

7 A. A. Brian and H. M. McConnell, Proc. Nat. Acad. Sci. USA, 1984, 81, 6159-6163.

8 V. I. Silin, H. Wieder, J. T. Woodward, G. Valincius, A. Offenhausser and A. L. Plant, J. Am. Chem. Soc., 2002, 124, 14676-14683.

9 E. Sackmann, Science., 1996, 271, 43-48.

10 H. Lang, C. Duschl and H. Vogel, Langmuir., 1994, 10, 197-210.

11 R. N. Orth, M. Wu, D. A. Holowka, H. G. Craighead and B. A. Baird, Langmuir., 2003, 19, 1599-1605.

12 M. Tanaka and E. Sackmann, Nature., 2005, 437, 656-663. 
13 J. T. Groves, Curr. Opin. Drug Discovery Dev., 2002, 5, 606-612.

14 S. Majd and M. Mayer, Angew. Chem. Int. Ed., 2005, 44, 6697-700.

15 J. T. Groves, N. Ulman, P. S. Cremer and S. G. Boxer, Langmuir., 1998, 14, 3347-3350.

16 K. Morigaki, T. Baumgart, A. Offenhäusser and W. Knoll, Angew. Chem. Int. Ed., 2001, 40, 172-174.

17 P. Kim, S. E. Lee, H. S. Jung, H. Y. Lee, T. Kawai and K. Y. Suh, Lab Chip., 2006, 6, 54-59.

18 L. A. Kung, L. Kam, J. S. Hovis and S. G. Boxer, Langmuir., 2000 , 16, 6773-6776.

19 V. Kiessling and L. K. Tamm, Biophys. J., 2003, 84, 408-418.

20 M. Tanaka and E. Sackmann, Nature., 2005, 437, 656-663.

21 B. A. Cornell, V. L. B. Braach-Maksvytis, L. G. King, P. D. J. Osman, B. Raguse, L. Wieczorek and R. J. A. Pace, Nature., 1997, 387, 580583.

22 M. L. Wagner and L. K. Tamm, Biophys. J., 2000, 79, 1400-1414.

23 M. Tanaka, A. P. Wong, F. Rehfeldt, M. Tutus and S. Kaufmann, J. Am. Chem. Soc., 2004, 126, 3257-3260.

24 F. Rehfeldt and M. Tanaka, Langmuir., 2003, 19, 1467-1473.

25 O. Purrucker, A. Förtig, K. Ludke, R. Jordan and M. Tanaka, J. Am. Chem. Soc., 2005, 127, 1258-1264.

26 A. T. A. Jenkins, R. J. Bushby, N. Boden, S. D. Evans, P. F. Knowles, Q. Liu, R. E. Miles and S. D. Ogier, Langmuir., 1998, 14, 4675-4678.
27 S. Chou, P. R. Krauss and P. J. Renstorm, Science, 1996, 272, 85-87. 28 J. Haisma, M. Verheijen, K. Vandenheuvel and J. Vandenberg, J. Vac. Sci. Technol. B, 1996, 14, 4124-236.

29 B. K. Lee, H. Y. Lee, P. Kim, K. Y. Suh, J. H. Seo, H. J. Cho and T. Kawai, Small., 2008, 3, 342-348.

30 P. Kim, B. K. Lee, H. Y. Lee, T. Kawai and K. Y. Suh, Adv. Mater., 2008, 20, 31-36.

31 D. A. Barrett, M. S. Hartshorne, M. A. Hussain, P. N. Shaw and M. C. Davies, Anal. Chem., 2001, 73, 5232-5239.

32 Y. Ito, M. Nogawa, M. Takeda and T. Shibuya, Biomaterials., 2005, 26, 211-216.

33 F. Albertorio, A. J. Diaz, T. Yang, V. A. Chapa, S. Kataoka, E. T. Castellana and P. S. Cremer, Langmuir., 2005, 21, 74767482.

34 Y. Cheng, N. Boden, R. J. Bushby, S. Clarkson, S. D. Evans, P. F. Knowles, A. Marsh and R. E. Miles, Langmuir., 1998, 14, 839-844.

35 L. J. C. Jeuken, S. D. Connell, M. Nurnabi, J. O'Reilly, P. J. F. Henderson, S. D. Evans and R. J. Bushby, Langmuir., 2005, 21, 1481-1488.

36 R. F. M. de Almeida, A. Fedorov and M. Prieto, Biophys. J., 2003, 85, 2406-2416.

37 J. C. Munro and C. W. Frank, Langmuir, 2004, 20, 3339-3349. 